\title{
OPTIMAL NON-COHERENT M-ARY ENERGY SHIFT KEYING FOR COOPERATIVE TRANSMISSION IN SENSOR NETWORKS
}

\author{
Albert Krohn \\ Telecooperation Office ( $\mathrm{TecO})$ \\ Institut für Telematik \\ Universität Karlsruhe
}

\begin{abstract}
Cooperative transmission follows the idea to achieve better reception quality for wireless communication systems by exploiting spatial diversity. We discuss the case where a group of sensor nodes transmit cooperatively and simultaneously identical symbols to a far destination. Doing so, they achieve a higher total transmit power. This is useful to reach destinations when no intermediate relays are able to act as repeater. We discuss this situation under the constraints of low power and low cost hardware and derive an optimal symbol constellation for a M-ary modulation scheme to minimize the symbol error. For this, we assume neither phase nor carrier synchronization between cooperatively transmitting nodes or the receiver yielding an easy to implement system with very low requirements for the participating wireless sensor nodes. Transmitted signals superimpose non-coherently in the receiver which uses only the amplitude information to decode symbols acting as an energy detector. We therefore name this modulation Energy Shift Keying (ESK).
\end{abstract}

\section{INTRODUCTION}

In wireless sensor networks, the quality of the channels and links between stations are often poor due to environmental factors such as occlusion, reflective objects, mobility and low power transmission. Figure 1 shows the typical application for cooperative transmission that we want to discuss as our reference scenario. Multi-hop connected sensor nodes distributed in the wilderness want to transmit collected data to a destination like a stationary antenna tower, plane or sattelite. The distance to the destination is too far, that a single node cannot communicate its data with sufficient SNR to the base station and there are no intermediate relays. This scenario has been previously named as the sensor reach back problem [1]. There are several arguments (e.g. the problem of a single point of failure) why a powerful up-link station among

Thanks to Dr. Karl Fabian (University Bremen, Germany) and Thomas Mautsch (ETH Zürich, Switzerland) for their helpful contribution in the analysis part of proof $A$

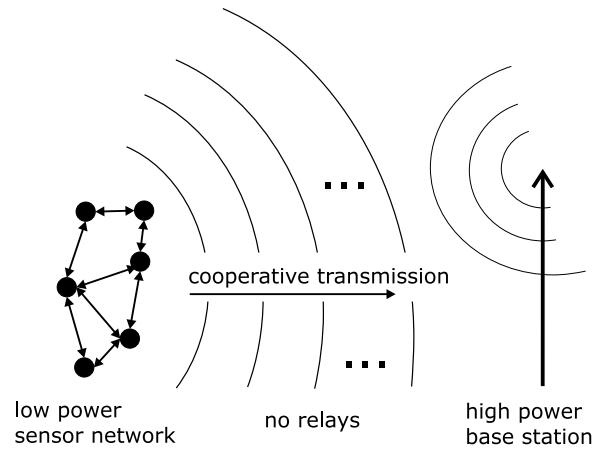

Fig. 1. Sensor Reach Back Problem

the sensor nodes is not expedient. We assume that time synchronization and random data exchange is possible between all pairs of nodes in the sensor network.

In this paper, we want to discuss cooperative transmission under the constraint of very cheap sensor network nodes to achieve a multistage (M-ary) modulation. Sensor nodes transmit data or symbols simultaneously over the radio channel to increase the total transmit power. Here, we find two major differences in the literature: Firstly, coherent cooperative transmission where stations superimpose their signals to achieve coherent phase in the destination receiver ([2], [3]) and secondly, non-coherent cooperative transmission where the station superimpose their signals without aligning their phases according to the receiver. The most related work can be found in [4] and [5]. In those publications, the authors understand cooperative transmission in the sense that several sensor nodes transmit symbols simultaneously to achieve a power gain. The authors propose a system using wide-band signals and derive an optimal receiver. We instead look at the transmitter and derive an optimal M-ary signal constellation for low-cost sensor nodes only using only time- synchronization, ASK in the sensor nodes and an energy-detector in the receiver. 


\section{NON-COHERENT COOPERATIVE TRANSMISSION}

For the system of cooperative transmission that we discuss in this paper, we assume that each of the $N$ transmitting nodes (denoted with index $l ; 1<l<N$ ) all have slightly different carrier frequencies $\omega_{l}=\omega_{c}+\Delta \omega_{l}$ with $\Delta \omega_{l}$ of unknown distribution due to production variation of the quartz oscillators. If the 1- th sensor node transmits a single complex symbol $S_{i}=a-j b$ (with $j$ as the imaginary unit, $0<i<M-1$ ), we get as transmitted signal in the time domain for the $1-$ th node:

$$
t_{l}(t)=\Re\left\{S_{i} \cdot w\left(t-\tau_{l}\right) \cdot e^{j\left(\omega_{l} t+\varphi_{l}\right)}\right\}
$$

With $\tau_{l}$ being the time delay or offset in symbol synchronization, $\omega_{l}$ the local carrier frequency, $\varphi_{l}$ the phase between receiver and the $l$-th transmitter and $w(t)$ the pulse shape window function. Looking at the single contribution term of one transmitter; after non-coherent (non-synchronized) down mix and low passing, the received signal of the 1-th transmitter without noise and channel influence is (in the baseband area of the receiver):

$$
\underline{r}_{l}(t)=w\left(t-\tau_{l}\right) \sqrt{a^{2}+b^{2}} \cdot e^{-j\left(\Delta \omega_{l} t+\varphi_{l}-\angle S_{i}\right)}
$$

Equation (2) shows a typical behavior of a non-coherent receiver. The received signal $\underline{r}_{l}(t)$ carries an oscillation with the frequency $\Delta \omega_{l}$. In contrast to [5], we do not consider this oscillation to be negligible. Taking e.g. a transceiver system on $2.4 \mathrm{GHz}$ with a quartz of 50ppm and symbol rates of some $\mathrm{kbit} / \mathrm{s}$, the $\Delta \omega_{l}$ is maximum $50 \mathrm{kHz}$ which is in the range of the symbol rate. In the receiver, we get a sum of multiple rotating signal points originated by many transmitters. We assume that a non-coherent reception can only use the received amplitude $u$ and/or the received differential phase of the received signal of which we pick only the amplitude. This has the interesting side effect that it is especially suitable and easy to realize in low cost hardware avoiding a complex modulation in the sensor nodes: they transmit using ASK.

\section{SYSTEM ASSUMPTIONS AND STATISTICS}

We summarize the assumption we will use for the next steps:

1. time delays $\tau_{l}$ are negligible $\left(\tau_{l}=0\right)$ as time- synchronization among the nodes is possible

2. phase shifts $\varphi_{l}$ are uniformly distributed over $[0 ; 2 \pi[$

3. frequency carrier offsets $\Delta \omega_{l}$ are unknown distributed

4. the channel is flat fading $g_{l}=\alpha e^{j \theta_{l}}$ with $0 \leq \alpha \leq 1$ and $\theta_{l}$ being uniformly distributed on $[0 ; 2 \pi[$. For simplicity we assume the fading $\alpha$ to be equal for all transmitters as the distances to the receiver are all nearly equal.

5. the noise is complex circular Gaussian $\sim \mathcal{N}\left(0, \sigma_{N}^{2} \mathbf{I}^{2}\right)$
6. all $M$ possible complex symbols $S_{i}$ have equal a-priori probability $P\left(S_{i}\right)=\frac{1}{M}$

For the derivation of the statistics of a single received signal/symbol we can apply the central limit theorem: For a high number of nodes simultaneously transmitting the same symbol received by a non-coherent receiver, we get multiple rotating signal points with nearly equal power (see assumptions above). The statistics of their sum will converge to a complex circular Gaussian distribution; the distribution of the amplitude will then be a rayleigh. It can be shown, that this approximation is already sufficient valid for a small number of nodes (typically 8). As the single contributions received by the receiver are i.i.d and the $\operatorname{cov}\left(\underline{r}_{l}, \underline{r}_{k}\right)=0 ; \forall l, k \in[1 ; N]$, we can simply add the variances and means of the $N$ received signals components on the I- $\left(\Re\left\{\underline{r}_{l}(t)\right\}\right)$ or the Q- $\left(\Im\left\{\underline{r}_{l}(t)\right\}\right)$ axis together. As the phase of the received signal points are uniformly distributed, the I,Q components of $\underline{r}(t)$ each carry half of the received power. Assuming equal receive power from all nodes (rayleigh approximation) and channel flat fading with scaling $\alpha$, the parameters for the received signal $\underline{r}(t)$ are:

$$
\begin{gathered}
\left(\begin{array}{c}
\mu_{\Im\{\underline{r}(t)\}} \\
\mu_{\Re\{\underline{r}(t)\}}
\end{array}\right)=N \cdot \underline{\mu}_{\underline{r}_{l}(t)}=\underline{0} \\
\sigma_{\Im\{\underline{r}(t)\}}^{2}=\sigma_{\Re\{\underline{r}(t)\}}^{2}=N \cdot \sigma_{\Re\left\{\underline{r}_{i}(t)\right\}}^{2}=N \alpha^{2} \sigma_{S_{i}}^{2}
\end{gathered}
$$

where we denote with $\sigma_{S_{i}}^{2}$ the transmit power on one component:

$$
\sigma_{S_{i}}^{2}:=\frac{1}{2}\left|S_{i}\right|^{2}
$$

The the marginal distribution over the amplitude (rayleigh) is:

$$
f_{S_{i}}(|\underline{r}(t)|=u)=\frac{u}{\left(N \alpha^{2} \sigma_{S_{i}}^{2}+\sigma_{N}^{2}\right)} e^{-\frac{u^{2}}{2\left(N \alpha^{2} \sigma_{S_{i}}^{2}+\sigma_{N}^{2}\right)}(5)}
$$

The transmitted symbols are only simple M-ASK symbols $S_{i}$ with powers $2 \sigma_{S_{i}}^{2}$. The receiver is an energy detector and we therefore call the modulation scheme ESK (energy shift keying). Hence forth, we base our discussion on (5) and summarize the term

$$
N \alpha^{2} \sigma_{S_{i}}^{2}+\sigma_{N}^{2}=: \sigma_{i}^{2}
$$

reflecting the fact that the statistics of the signals and the noise are both complex circular Gaussian.

\section{OPTIMAL SIGNAL CONSTELLATION FOR M-ARY ENERGY SHIFT KEYING (ESK) TO ACHIEVE MINIMUM TOTAL ERROR}

We are now interested in how the symbols $S_{i}$ (or more precisely: the power levels $\sigma_{S_{i}}^{2}$ ) should be chosen to arrive at an optimal constellation for the energy-detection. We call $H_{i}$ the 
decision of the receiver for signal $S_{i}$. We consider as optimality criterion that the symbols $S_{i}$ are chosen in a way that the detection rate for all symbols are maximal:

$$
P=\sum_{i=0}^{M-1} P\left(S_{i}\right) P\left(H_{i} \mid S_{i}\right) \rightarrow \max
$$

For the derivation of the optimal symbol constellation under condition (7), the optimal decision thresholds for an optimal detector (Maximum Likelihood) lies at the intersection of the neighbouring rayleigh distributions. Using (6) in (5), this intersection of two distributions with $\sigma_{i}^{2}$ and $\sigma_{i+1}^{2}$ is:

$$
\begin{aligned}
\frac{u}{\sigma_{i}^{2}} e^{-\frac{u^{2}}{2 \sigma_{i}^{2}}} & =\frac{u}{\sigma_{i+1}^{2}} e^{-\frac{u^{2}}{2 \sigma_{i+1}^{2}}} \\
\Leftrightarrow u^{2} & =\ln \left(\frac{\sigma_{i}^{2}}{\sigma_{i+1}^{2}}\right) \cdot \frac{2 \sigma_{i}^{2} \sigma_{i+1}^{2}}{\sigma_{i}^{2}-\sigma_{i+1}^{2}}=: \gamma_{i \rightarrow i+1}^{2}
\end{aligned}
$$

The detection probability (7) then is:

$$
\begin{gathered}
P=\frac{1}{M} \sum_{i=0}^{M-1} \int_{\gamma_{i-1 \rightarrow i}}^{\gamma_{i \rightarrow i+1}} f_{\sigma_{i}^{2}}(u) d u \\
\text { with } f_{\sigma_{i}^{2}}(u)=\frac{u}{\sigma_{i}^{2}} e^{-\frac{u^{2}}{2 \sigma_{i}^{2}}}
\end{gathered}
$$

Applying (8) leads to

$$
\begin{aligned}
M \cdot P=P_{d} & =1-e^{\frac{\ln \left(\frac{\sigma_{1}^{2}}{\sigma_{2}^{2}}\right) \cdot \sigma_{1}^{2}}{\sigma_{0}^{2}-\sigma_{1}^{2}}}+ \\
& +\sum_{i=0}^{M-3}\left[e^{\frac{\ln \left(\frac{\sigma_{i+1}^{2}}{\sigma_{i}^{2}}\right) \cdot \sigma_{i}^{2}}{\sigma_{i}^{2}-\sigma_{i+1}^{2}}}-e^{\frac{\ln \left(\frac{\sigma_{i+2}^{2}}{\sigma_{i+1}^{2}}\right) \cdot \sigma_{i+2}^{2}}{\sigma_{i+1}^{2}-\sigma_{i+2}^{2}}}\right]+ \\
& +e^{\frac{\ln \left(\frac{\sigma_{M-1}^{2}}{\sigma_{M-2}^{2}}\right) \cdot \sigma_{M-2}^{2}}{\sigma_{M-2}^{2}-\sigma_{M-1}^{2}}}
\end{aligned}
$$

$\sigma_{0}^{2}$ and $\sigma_{M-1}^{2}$ are not subject to optimization as they are set to the minimum and maximum powers derived from the system energy constraints with $\sigma_{0}^{2}=0$ and $\sigma_{M-1}^{2}=$ the maximum transmit power. With the help of the $\sigma_{0}^{2}=0$ and the virtual signal power $\sigma_{M}^{2}=\infty$, the expression (10) can be written in an more compact form:

$$
P_{d}=\sum_{i=0}^{M-2}\left[e^{\frac{\ln \left(\frac{\sigma_{i+1}^{2}}{\sigma_{i}^{2}}\right) \cdot \sigma_{i}^{2}}{\sigma_{i}^{2}-\sigma_{i+1}^{2}}}-e^{\frac{\ln \left(\frac{\sigma_{i+2}^{2}}{\sigma_{i+1}^{2}}\right) \cdot \sigma_{i+2}^{2}}{\sigma_{i+1}^{2}-\sigma_{i+2}^{2}}}\right]
$$

The expression (11) must be maximized by choosing the optimal $\sigma_{i}^{2} ; 1<i<M-2$. Therefore, We take out all contribution of (10) with terms containing $\sigma_{i}^{2}$ and understand $\sigma_{i-1}^{2}$ and $\sigma_{i+1}^{2}$ as parameters:

$$
\begin{aligned}
P_{d, \sigma_{i-1}^{2}, \sigma_{i+1}^{2}}\left(\sigma_{i}^{2}\right)= & -e^{\frac{\ln \left(\frac{\sigma_{i}^{2}}{\sigma_{i-1}^{2}}\right) \cdot \sigma_{i}^{2}}{\sigma_{i-1}^{2}-\sigma_{i}^{2}}}+e^{\frac{\ln \left(\frac{\sigma_{i}^{2}}{\sigma_{i-1}^{2}}\right) \cdot \sigma_{i-1}^{2}}{\sigma_{i-1}^{2}-\sigma_{i}^{2}}} \\
& -e^{\frac{\ln \left(\frac{\sigma_{i+1}^{2}}{\sigma_{i}^{2}}\right) \cdot \sigma_{i+1}^{2}}{\sigma_{i}^{2}-\sigma_{i+1}^{2}}}+e^{\frac{\ln \left(\frac{\sigma_{i+1}^{2}}{\sigma_{i}^{2}}\right) \cdot \sigma_{i}^{2}}{\sigma_{i}^{2}-\sigma_{i+1}^{2}}}
\end{aligned}
$$

One can see that this expression only depends on $\sigma_{i-1}^{2}, \sigma_{i}^{2}$ and $\sigma_{i+1}^{2}$. We can therefore take the local maximum solutions and combine them in a vector maximizing (11). We prove in Appendix A that the optimal signal constellation (with respect to minimum total error) is achieved, when the powers of the received symbols fulfill the iterative condition:

$$
\sigma_{i}^{2}=k \cdot \sigma_{i-1}^{2} \quad \text { with } 1<i<M ; \quad k>1, k \in \mathrm{R}
$$

For the optimal signal constellation, we apply (6) in (13) and resolve for a rule how the transmitters should chose their symbols:

$$
\sigma_{S_{i}}^{2}=\frac{k\left(\sigma_{N}^{2}+N \alpha^{2} \sigma_{S_{i-1}}^{2}\right)-\sigma_{N}^{2}}{N \alpha^{2}}
$$

For this solution it is required that the transmitters know the number of nodes $N$ and the noise level in the receiver, which is not automatically the case. But we can simplify (14) if we assume $\sigma_{S_{M-1}}^{2} \gg \sigma_{S_{0}}^{2} \gg \sigma_{N}^{2}$ (and do not choose $\sigma_{0}^{2}=0$ !):

$$
\sigma_{S_{i}}^{2} \approx k \cdot \sigma_{S_{i-1}}^{2} \quad \text { and } \quad k \approx \sqrt[(M-1)]{\frac{\sigma_{S_{M-1}}^{2}}{\sigma_{S_{0}}^{2}}}
$$

\section{CONCLUSION}

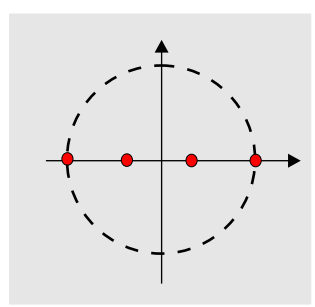

4- ASK

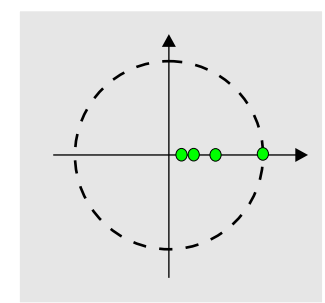

4- ESK
Fig. 2. Example signal constellation in the I-, Q-plane for traditional (left) and cooperative (right) transmission

With (14), we derived the optimal signal constellation for an $\mathrm{M}$-ary signal constellation using non-coherent cooperative transmission. Our optimality criterion (7) for minimal symbol error was accompanied with the contraint of a given maximal power for $\sigma_{M-1}^{2}$, shown as the dotted line in figure 2. Further, with (15), we give a practical approximation. In figure 
2, we illustrate an example result of a 4-ESK compared to a traditional 4-ASK. Using these ESK- symbols and a noncoherent cooperative transmission, the far receiver has an optimal symbol constellation when using an ML- energy detector. It is also important to remember, that we don't assume the oscillations to be negligible during the duration of one symbol. These oscillations cause the rayleigh-distribution of the received amplitude (5) of one symbol as a result of many superimposed statistics.

\section{ACKNOWLEDGMENT}

The work presented in this paper was partially funded by the European Community through the projects CoBIs (contract no. 4270) and RELATE and by the Ministry of Economic Affairs of the Netherlands through the BSIK project Smart Surroundings under contract no. 03060.

\section{REFERENCES}

[1] J. Barros and S. D. Servetto, "Network information flow with correlated sources," in (Submitted to the IEEE Transactions on Information Theory).

[2] Y.-S. Tu and G. Pottie, "Coherent cooperative transmission from multiple adjacent antennas to a distant stationary antenna through awgn channels," in Proceeding of the IEEE VTC Spring 02, Birmingham, Alabama, USA, May 2002.

[3] D. III, G.Prince, and J. McNeill, "Method for carrier frequency and phase synchronization of two autonomous cooperative transmitters," in 5th IEEE Signal Processing Advances in Wireless Communications (SPAWC) 2005.

[4] A. Scaglione and Y.-W. Hong, "Opportunistic large arrays: Cooperative transmission in wireless multihop ad hoc networks to reach far distances," IEEE Transaction on Signal Processing, vol. 51, no. 8, August 2003.

[5] B. Sirkeci-Mergen and A. Scaglione, "Signal acquisition for cooperative transmissions in multi-hop ad-hoc networks," in Proceeding of the International Conference on Acoustics, Speech, and Signal Processing (ICASSP), Montreal, Canada, May 2004.

\section{A. APPENDIX}

To find the global maximum of (11), we substitute:

$$
\begin{aligned}
& a=\sqrt{\frac{\sigma_{i+1}^{2}}{\sigma_{i-1}^{2}}} \text { the arbitrary parameter } \\
& x=\sqrt{\frac{\sigma_{i}^{2} \sigma_{i}^{2}}{\sigma_{i-1}^{2} \sigma_{i+1}^{2}}} \quad \text { the new variable }
\end{aligned}
$$

A general condition is that $\sigma_{i-1}^{2}<\sigma_{i}^{2}<\sigma_{i+1}^{2}$ and with (16) and (17) we see that $\frac{1}{a}<x<a$. Further, with $\sigma_{i+1}^{2} \geq \sigma_{i}^{2}$ follows: $a>1$. With (16), (17) in (12) we define:

$$
\begin{aligned}
g_{a}(x) & :=-e^{\frac{\ln (a x)}{a x}-1}+e^{\frac{\ln (a x)}{1-a x}}-e^{\frac{\ln \left(\frac{a}{x}\right)}{\frac{x}{a}-1}}+e^{\frac{\ln \left(\frac{a}{x}\right)}{1-\frac{a}{x}}} \\
f(y) & :=y^{\frac{1}{1-y}}-\frac{1}{y} \cdot y^{\frac{1}{1-y}} \\
& \Rightarrow g_{a}(x)=f(a x)+f\left(\frac{a}{x}\right) \\
q(y) & :=\frac{\partial f(y)}{\partial y} \cdot y=y^{\frac{-1}{y-1}} \frac{\ln y}{y-1}
\end{aligned}
$$

so that

$$
\frac{d f(a x)}{d x}=q(a x) \frac{1}{x} \text { and } \quad \frac{d f\left(\frac{a}{x}\right)}{d x}=-q\left(\frac{a}{x}\right) \frac{1}{x}
$$

and

$$
\frac{d g_{a}(x)}{d x}=\frac{1}{x}\left(q(a x)-q\left(\frac{a}{x}\right)\right)
$$

It can be shown that $q(y)$ is a strictly monotonic decreasing function for $y>1$ :

$$
q^{\prime}(y)=\underbrace{y^{\frac{-1}{y-1}}}_{>0} \underbrace{>0}_{=: r(y)} \frac{\overbrace{\frac{1}{y(y-1)^{3}}}^{\left((y-1)^{2}-\left(y^{2}-1\right) \ln y+y \ln ^{2} y\right)}}{(y-1)}
$$

and $r(y)<0$ for $y>1$, as

$$
r(1)=\frac{d r}{d y}(1)=\frac{d^{2} r}{d y^{2}}(1)=0
$$

and

$$
\frac{d^{3} r}{d y^{3}}=\underbrace{\frac{-2}{y^{3}}}_{<0} \underbrace{\left(y^{2}-1+y \ln y\right)}_{=: s(y)}
$$

and $s(1)=0, s^{\prime}(y)=2 y+\ln y+1>0$ for $y>1$. Knowing $q^{\prime}(y)<0$ for $y>1$ and going back to (21) and (22), we see:

$$
\begin{array}{ll}
x>1 \quad: \quad a x>\frac{a}{x} \Rightarrow q(a x)<q\left(\frac{a}{x}\right) \Rightarrow \frac{d g_{a}}{d x}<0 \\
x<1: \quad a x<\frac{a}{x} \Rightarrow q(a x)>q\left(\frac{a}{x}\right) \Rightarrow \frac{d g_{a}}{d x}>0 \\
x=1 \quad: \quad a x=\frac{a}{x} \Rightarrow \frac{d g_{a}}{d x}=0
\end{array}
$$

meaning that $x=1$ is the global maximum of $g_{a}(x)$. Reapplying $x=1$ in (17) results in :

$$
\frac{\sigma_{i+1}^{2}}{\sigma_{i}^{2}}=\frac{\sigma_{i}^{2}}{\sigma_{i-1}^{2}}
$$

considering that $\sigma_{i}^{2}>\sigma_{i-1}^{2}$, we find (13) to fulfil (24). 